\title{
LOW- $x$ HADRONIC FINAL STATES AT HERA
}

\author{
MICHAEL KUHLEN \\ Max-Planck-Institut für Physik, Werner-Heisenberg-Institut \\ Föhringer Ring 6, D-80805 München, Germany \\ E-mail: kuhlen@desy.de
}

representing the $\mathrm{H} 1$ and ZEUS Collaborations at the Madrid Workshop on low- $x$ Physics, Miraflores de la Sierra, June 1997

\begin{abstract}
Measurements of the hadronic final state at HERA are reviewed, which aim at the investigation of the parton dynamics of the proton at small Bjorken $x$.
\end{abstract}

\section{Introduction}

Amongst the most interesting issues of HERA physics is QCD in the newly accessible regime of small Bjorken $x$. The observed rise of the structure function $F_{2}$ towards small $x$ suggests a strong increase of the parton density in the proton, but what is its dynamical origin? Is BFKL 1] dynamics at work, or is conventional DGLAP [2] evolution sufficient? Complementary measurements of the hadronic final state provide more detailed information than the inclusive $F_{2}$ data to help uncover the underlying dynamics.

The leading log DGLAP resummation corresponds to a strong ordering of the transverse momenta $k_{T}$ (w.r.t. the proton beam) in the parton cascade,

a)

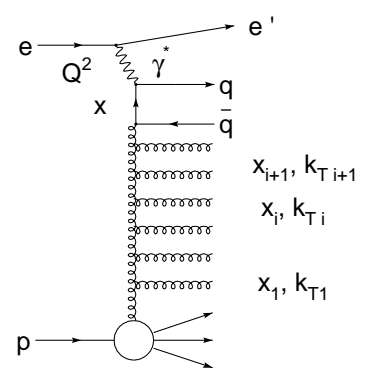

Figure 1: a) Ladder diagram for parton evolution. b) The multiplicity of hard gluons with $p_{T}>2 \mathrm{GeV}$ vs. CMS $\eta$. The events are generated with the models CDM, MEPS and HERWIG in a "low $x$ " kinematic bin with $\langle x\rangle=0.00037$ and $\left\langle Q^{2}\right\rangle=13.1 \mathrm{GeV}^{2}$. The proton direction is to the left.

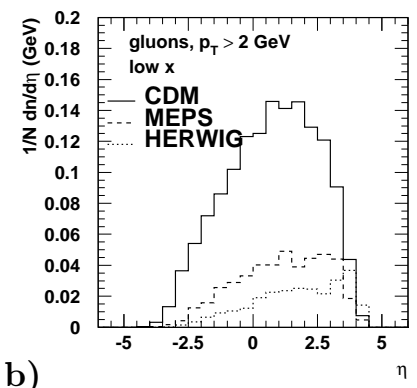

b) 
$Q_{0}^{2} \ll k_{T 1}^{2} \ll \ldots k_{T i}^{2} \ll \ldots Q^{2}$ (fig. 17a). Since in the BFKL evolution that restriction is absent (" $k_{T}$ diffusion"), a generic signal for deviations from DGLAP evolution is enhanced radiation from the ladder between the current and the remnant system, that is in the central rapidity region in the hadronic centre of mass system (CMS). Experimentally pseudorapidity $\eta=-\ln \tan (\theta / 2)$ is used, where $\theta$ is the angle with respect to the virtual photon axis. The following observables have so far been exploited and are being discussed in this paper: 1) $E_{T}$ flows: increased parton activity should result in an increased transverse energy flow [3]. 2) Charged particle $p_{T}$ spectra: high $k_{T}$ partons, disfavoured by the strong $k_{T}$ ordering in DGLAP, are signalled by measureable high $p_{T}$ hadrons [4]. 3) Forward jets: high energy jets with $p_{T \text { jet }}{ }^{2} \approx Q^{2}$ (kinematically bound to be measured in the forward calorimetric systems close to the remnant direction) tag events with BFKL evolution, because DGLAP evolution is not allowed [5, 6].

Theoretical calculations for these observables exist and can be compared to the data, provided that hadronization effects can be controlled. Alternatively, predictions are derived from Monte Carlo models, which incorporate the QCD evolution in different approximations and utilize phenomenological models for the non-perturbative hadronization phase. The MEPS model (Matrix Element plus Parton Shower, program LEPTO [7]) and the HERWIG generator [8] are based upon leading log DGLAP parton showers, with strong $k_{T}$ ordering of the emitted partons. In the colour dipole model (CDM) [9, 10] gluon emission is not subject to $k_{T}$ ordering [11]. In that respect it mimicks the BFKL evolution, and leads to more abundant gluon radiation than in the other models (fig. 1 $1 \mathrm{~b}$ ).

\section{Energy flows}

The flow of transverse energy $E_{T}$ as a function of $\eta$ provides a very simple, global characterization of the hadronic final state. Though their partonic radiation patterns are very different, all models in their present incarnations provide a reasonable description of the detailed $E_{T}$ flow data by H1 [12] (two out of $17 x-Q^{2}$ bins are shown in fig. 2).

From BFKL evolution a relatively large amount of $E_{T}$ at central rapidity is expected that increases with decreasing $x$, opposite to the DGLAP expectation [3]. These behaviours are realized at the parton level for the models with the unordered and ordered emission scenarios (fig. 3). The average $E_{T}\left(\left\langle E_{T}\right\rangle\right)$ measured in $-0.5<\eta<0.5$ [12, 13] does increase with falling $x$, in qualitative agreement with the BFKL expectation (fig. 3). However, when comparing to the calculation hadronization effects have to be taken into account.

The DGLAP based models have to employ large hadronization corrections 


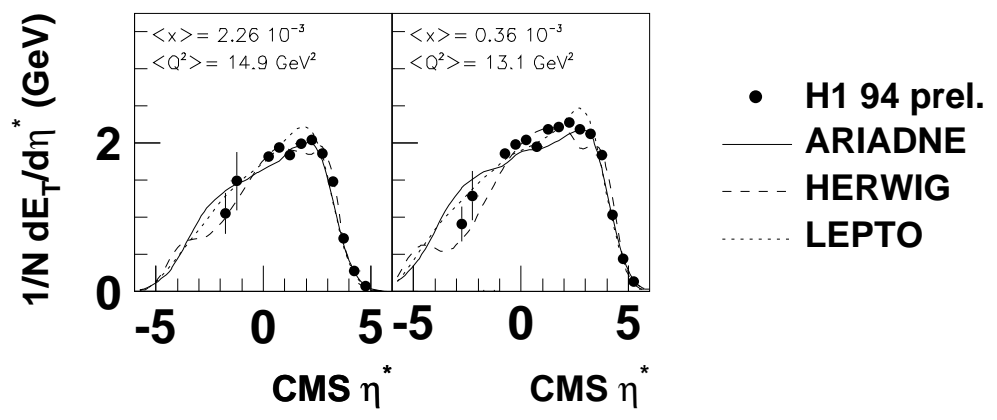

Figure 2: The $E_{T}$ flow vs. $\eta$ in the hadronic CMS for $\langle x\rangle \approx 0.002$ and $\langle x\rangle \approx 0.0004$ with $\left\langle Q^{2}\right\rangle \approx 14 \mathrm{GeV}^{2}$ fixed. The proton direction is to the left. The data 12 are compared to the models CDM (ARIADNE 4.08), MEPS (LEPTO 6.4), and HERWIG 5.8.

to achieve the level of $E_{T}$ seen in the data (see fig. $3 \mathrm{~b}$ ). For example, in LEPTO the new concept of soft colour interactions (SCI) has been introduced, resulting in a reconfiguration of the fragmenting strings, that may lead to an enhancement of $E_{T}$ (and also to rapidity gaps) 14. In the CDM, hadronization effects are much smaller than in LEPTO and HERWIG (fig. 3). In summary, though the data follow the trend expected from BFKL evolution and are consistent with a model with an unsuppressed radiation scenario, they are also consistent with DGLAP evolution, assuming large hadronization effects.

\section{Charged particle transverse momenta}

Not yet well understood hadronization effects precluded strong conclusions on the underlying parton dynamics from the $E_{T}$ flow measurements. Single particle transverse momentum $\left(p_{T}\right)$ spectra represent a more direct measure of the partonic activity [ [- 1 . Unsuppressed parton radiation should manifest itself in a hard tail of the $p_{T}$ distribution, whereas hadronization should produce typical spectra limited in $p_{T}$.

H1 has measured the charged particle $p_{T}$ spectra as much central in the CMS $(0.5<\eta<1.5)$ as the tracking detector acceptance allowed 15]. At large $x$, all models agree with the data, but at small $x$, only the model with the unsuppressed radiation pattern $(\mathrm{CDM})$ is able to describe the high $p_{T}$ tail seen in the data (fig. (1) The shortfall of the models with suppressed gluon radiation indicates that at small $x$ there is more high $k_{T}$ parton radiation present than is produced by the models based upon leading log DGLAP parton showers. 


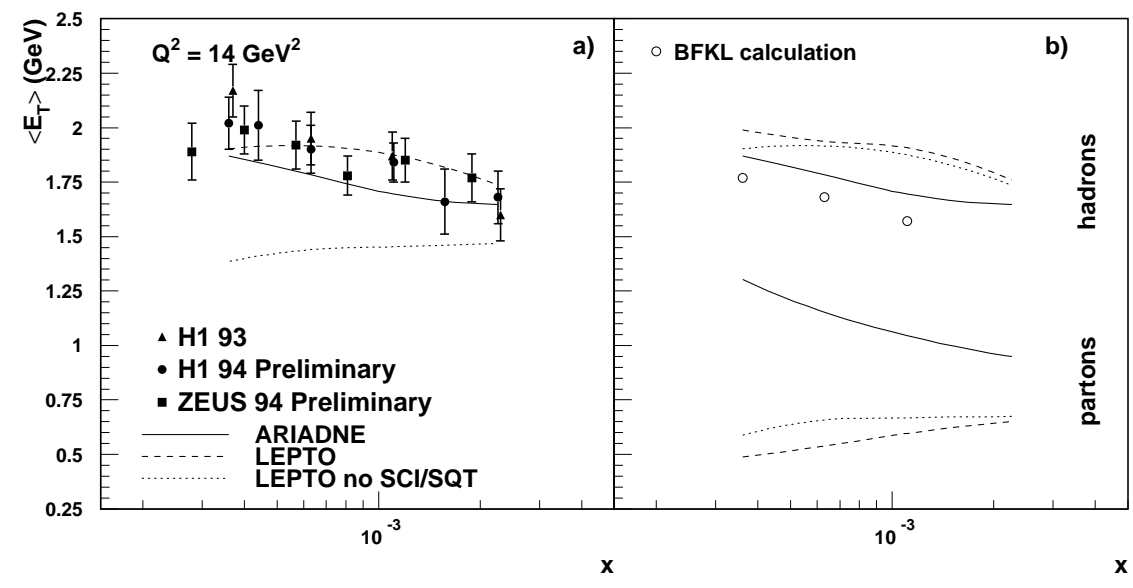

Figure 3: The mean $E_{T}(\mathrm{GeV})$ in $-0.5<\eta<0.5$ as a function of $x$ for $Q^{2}=14 \mathrm{GeV}^{2}$. The data 12, 13] are shown together with the models CDM (ARIADNE 4.08), MEPS (LEPTO 6.4), and HERWIG 5.8 for hadrons and for partons, and with the BFKL calculation for partons [3]. The LEPTO result for hadrons without the features soft colour interaction (SCI) and the new sea quark treatment (SQT) is also shown.

The $p_{T}$ spectra at small $x$ have been calculated by folding a cross section $\sigma_{j}$ to produce a parton $j$ with experimentally known fragmentation functions $D_{h / j}(z)$ to produce a hadron $h$ with momentum fraction $z$ from the parton $j$ [16]. Symbolically, $\sigma_{h}=\sigma_{j} \otimes D_{h / j}$. Monte Carlo assumptions for hadronization are thus avoided. When BFKl evolution is invoked, the $\mathrm{H} 1$ data are well described by the calculation (fig $4 \mathrm{~b}$ ). The normalization for the BFKL part is obtained by requiring that the calculated parton cross section would match the measured H1 forward hadron jets 117 (see next sect.). Neglecting BFKL evolution, the calculation falls significantly below the data. It would now be interesting to compare a complete fixed order calculation in next-to-leading order (NLO) to the data.

\section{Forward jets}

Forward jets are the classic signature for BFKL evolution [5, 6]. One requires $x_{\text {jet }}=E_{\text {jet }} / E_{p}$, the ratio of jet and proton energy to be large in order to maximize the BFKL evolution from $x_{\text {jet }}$ down to $x$. The jet transverse momentum $k_{T \text { jet }}$ has to be close to $Q, k_{T \text { jet }} \approx Q$ to suppress the phase space for DGLAP 

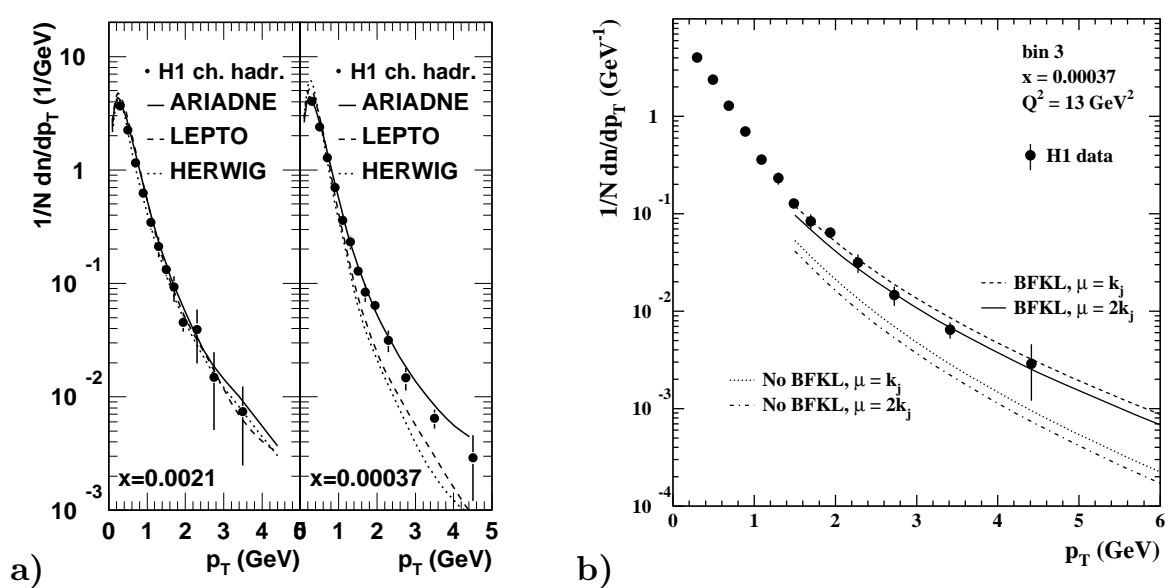

Figure 4: a) The $p_{T}$ spectra of charged particles from $0.5<\eta<1.5$ (CMS) 15 . Displayed are two different kinematic bins at high and low $x$ for $\left\langle Q^{2}\right\rangle \approx 14 \mathrm{GeV}^{2}$. The models ARIADNE 4.8, LEPTO 6.4 and HERWIG 5.8 are overlayed. b) The result of a theoretical calculation with and without BFKL evolution [16], compared to the H1 data at low $x$ for different choices of the factorization scale $\mu$.

evolution. In the presence of BFKL evolution, the forward jet cross section should grow faster with decreasing $x$ than for DGLAP evolution.

$\mathrm{H} 1$ reconstructs forward jets with the cone algorithm and requires $p_{T \text { jet }}>$ $3.5 \mathrm{GeV}$ [17. The forward jet cross section (fig. Fa), corrected for detector effects, is well described by the CDM, and somewhat less well by the standard MEPS model with SCI (note that SCI was necessary in order to describe the $E_{T}$ flows). Without SCI, the DGLAP based model MEPS cannot describe the growth of the cross section towards small $x$. Comparing the hadron level jets to the parton level jets (fig. $5 \mathrm{~b}$ ), one finds relatively small hadronization corrections in the CDM and also in MEPS without SCI, but with SCI, hadronization effects become large! If one would assume the CDM hadronization corrections to be correct, the data would be far above both the NLO calculation [18] and the parton level jets from the DGLAP based model MEPS.

Presumably hadronization uncertainties become smaller for a higher $p_{T \text { jet }}$ cut-off. In the ZEUS analysis [20, which relies also on the cone algorithm, $p_{T \text { jet }}>5 \mathrm{GeV}$ is required. The data in fig. Fc are corrected to the parton level, assuming hadronization corrections from the CDM. In contrast to a NLO jet calculation [21], the CDM describes the data well.

It is clear that in order to draw firm conclusions from the forward jet 

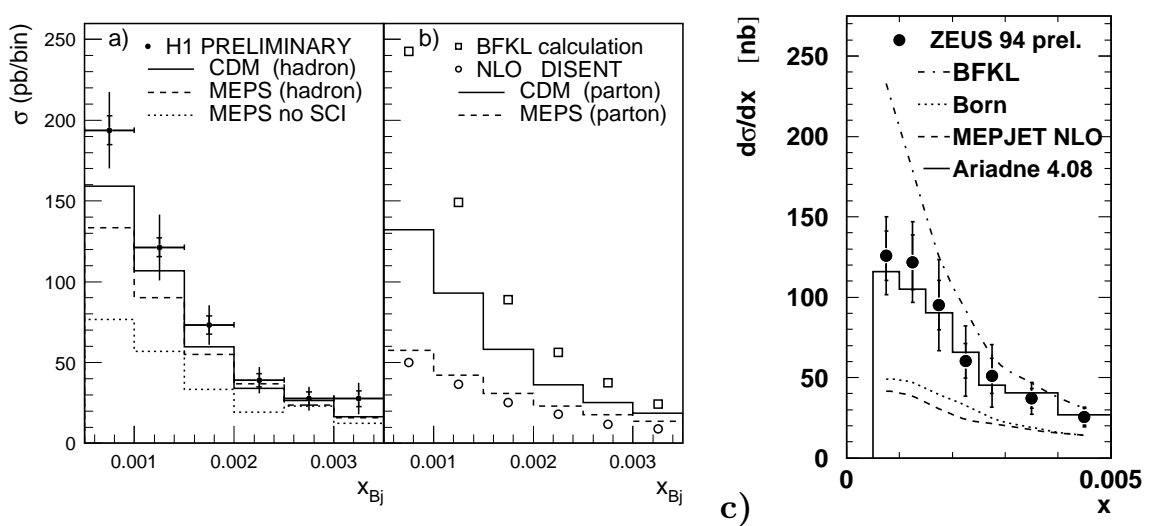

Figure 5: a) The H1 forward jet cross section 17] (1994 data) vs. $x$, corrected for detector effects to the hadron level. $x_{\text {jet }}>0.035,0.5<p_{T \text { jet }}{ }^{2} / Q^{2}<2$ and $p_{T \text { jet }}>3.5 \mathrm{GeV}$ were required. Also shown are the hadron level predictions from CDM (ARIADNE 4.08) and MEPS (LEPTO 6.4, with and without SCI). b) The corresponding parton level forward jet cross section from a NLO calculation [18], and for CDM and MEPS. Also shown is a BFKL parton cross section calculation 19] (no jet algorithm). c) The ZEUS forward jet cross section 20] (1994 data) vs. $x$, corrected to the parton level. $x_{\text {jet }}>0.035,0.5<p_{T \text { jet }}{ }^{2} / Q^{2}<4$ and $p_{T \text { jet }}>5 \mathrm{GeV}$ were required. The data are compared to a NLO jet calculation [21] and to parton jets from CDM (ARIADNE 4.08). Also shown are parton cross sections (no jet algorithm) with ("BFKL") and without ("Born") BFKL evolution [19]. The systematic errors do not include uncertainties due to hadronization.

analyses, hadronization effects need to be better understood. It is however interesting to note that the BFKL calculation [19] is far above the Born level calculation (excluding BFKL evolution), and describes qualitatively the rise of the measured forward jet cross sections at small $x$ (fig. [). Direct comparison to the data however would be imprudent, because these calculations do not invoke a jet algorithm, and hadronization corrections are potentially large.

\section{Conclusion}

The transverse energy flow and the forward jet data are only compatible with a conventional DGLAP evolution scenario when large hadronization effects are assumed. The high $p_{T}$ tail seen in the charged particle spectra cannot be explained with hadronization effects. A theoretical calculation neglecting BFKL evolution, where hadronization is taken into account via known fragmentation 
functions, falls far below the data. The $p_{T}$ spectra require more hard parton radiation than expected from conventional DGLAP evolution. BFKL effects offer a consistent explanation of the measured $p_{T}$ spectra, forward jets and $E_{T}$ flows. Of course this does not exclude other explanations. For example, it has been suggested that contributions from resolved virtual photons could be responsible for enhanced parton activity 22. In that case the hard scattering takes place between partons inside the resolved photon and partons from the proton and may thus happen not at the photon vertex but further down the ladder.

\section{References}

1. E.A. Kuraev, L.N. Lipatov and V.S. Fadin, Sov. Phys. JETP 45 (1972) 199; Y.Y. Balitsky and L.N. Lipatov, Sov. J. Nucl. Phys. 28 (1978) 282.

2. Yu. L. Dokshitzer, Sov. Phys. JETP 46 (1977) 641;

V.N. Gribov and L.N. Lipatov, Sov. J. Nucl. Phys. 15 (1972) 438 and 675;

G. Altarelli and G. Parisi, Nucl. Phys. 126 (1977) 297.

3. J. Kwieciński, A.D. Martin, P.J. Sutton and K.Golec-Biernat, Phys. Rev. D50 (1994) 217; Phys. Lett. B335 (1994) 220.

4. M. Kuhlen, Phys. Lett. B382 (1996) 441; MPI-PhE/96-18, hep-ex/9610004.

5. A.H. Mueller, Nucl. Phys. B (Proc. Suppl.) 18C (1990) 125; J. Phys. G17 (1991) 1443.

6. J. Bartels, A. De Roeck, M. Loewe, Z. Phys. C54 (1992) 635;

W.K. Tang, Phys. Lett. B278 (1992) 363.

7. G. Ingelman, Proc. Workshop on Physics at HERA, Hamburg 1991, eds. W. Buchmüller and G. Ingelman, vol. 3, p. 1366;

G. Ingelman, A. Edin and J. Rathsman, Comp. Phys. Comm. 101 (1997) 108.

8. G. Marchesini et al., Comp. Phys. Comm. 67 (1992) 465 .

9. G. Gustafson, Ulf Petterson, Nucl. Phys. B306 (1988);

G. Gustafson, Phys. Lett. B175 (1986) 453;

B. Andersson, G. Gustafson, L. Lönnblad, Ulf Petterson, Z. Phys. C43 (1989) 625 .

10. L. Lönnblad, Comp. Phys. Comm. 71 (1992) 15.

11. L. Lönnblad, Z. Phys. C65 (1995) 285; CERN-TH/95-95;

A. H. Mueller, Nucl. Phys. B415 (1994) 373 .

12. H1 Collab., contrib. paper pa02-073 to ICHEP'96, Warsaw 1996.

13. N. Pavel for the ZEUS Collab., Proc. DIS96 workshop, Rome 1996.

14. A. Edin, G. Ingelman and J. Rathsman, DESY 96-019; Phys.Lett. B366 (1996) 371; Z. Phys. C75 (1997) 57.

15. H1 Collab., C. Adloff et al., Nucl. Phys. B485 (1997) 3.

16. J. Kwieciński, S.C. Lang and A.D. Martin, DTP/97/56, hep-ph/9707240.

17. H1 Collab., contrib. paper pa03-049 to ICHEP'96, Warsaw 1996.

18. S. Catani, M. Seymour, Phys. Lett. B378 (1996) 287 , Nucl. Phys. B485 (1997) 291.

19. J. Bartels et al., Phys. Lett. B384 (1996) 300.

20. S. Wölfle for the ZEUS Collab., DIS97 workshop, Chicago 1997.

21. T. Brodkorb and E. Mirkes, Z. Phys. C66 (1995) 141.

22. H. Jung, these Proceedings. 